\title{
Blocking IL-17A Alleviates Diabetic Retinopathy in Rodents
}

\author{
Ao-Wang Qiu Jun-Ling Wang Qing-Huai Liu \\ Department of Ophthalmology, The First Affiliated Hospital of Nanjing Medical University, Nanjing, China
}

\section{Key Words}

Interleukin-17A • Interleukin-17 receptor A • Diabetic retinopathy • Retinal Müller cells

\begin{abstract}
Background/Aims: Interleukin (IL)-17A, a proinflammatory cytokine, has been implicated in several autoimmune diseases. However, it is unclear whether IL-17A is involved in diabetic retinopathy (DR), one of the most serious complications of autoimmune diabetes. This study aimed to demonstrate that IL-17A exacerbates DR by affecting retinal Müller cell function. Methods: High glucose (HG)-treated rat Müller cell line (rMC-1) was exposed to IL-17A, antiIL-17A-neutralizing monoclonal antibody ( $\mathrm{mAb}$ ) or/and anti-IL-17 receptor (R)A-neutralizing $\mathrm{mAb}$ for $24 \mathrm{~h}$. For in vivo study, DR was induced by intraperitoneal injections of streptozotocin (STZ). DR model mice were treated with anti-IL-17A mAb or anti-IL-17RA mAb in the vitreous cavity. Mice that were prepared for retinal angiography were sacrificed two weeks after intravitreal injection, while the rest were sacrificed two days after intravitreal injection. Results: IL-17A production and IL-17RA expression were increased in both HG-treated $\mathrm{rMC}-1$ and DR retina. HG induced $\mathrm{rMC}-1$ activation and dysfunction, as determined by the increased GFAP, VEGF and glutamate levels as well as the downregulated GS and EAAT1 expression. IL-17A exacerbated the HG-induced $\mathrm{rMC}-1$ functional disorders, whereas either anti-IL-17A mAb or anti-IL-17RA mAb alleviated the HG-induced $\mathrm{rMC}-1$ disorders. Intravitreal injections with anti-IL-17A mAb or anti-IL-17RA mAb in DR model mice reduced Müller cell dysfunction, vascular leukostasis, vascular leakage, tight junction protein downregulation and ganglion cell apoptosis in the retina. Conclusions: IL-17A aggravates DR-like pathology at least partly by impairing retinal Müller cell function. Blocking IL-17A is a potential therapeutic strategy for DR.

\section{Introduction}

Interleukin (IL)-17, a proinflammatory cytokine, is principally produced by T-helper (Th)17 cells. The IL-17 cytokine family consists of six cytokines (IL-17A-IL-17F) and five receptors (IL-17RA-IL-17RE) [1]. IL-17A is one particular cytokine that has raised substantial interest in the recent years, as it has been implicated in several autoimmune diseases, such as rheumatoid arthritis, psoriasis, and multiple sclerosis [2, 3]. However, still

Qing-Huai Liu

KARGER
Department of Ophthalmology, the First Affiliated Hospital of Nanjing Medical University, 300 Guangzhou Road, Nanjing, Jiangsu Province 210029, (China)

Tel. +86-25-83714511, E-Mail qh_liu@yeah.net / liuqh@njmu.edu.cn 
very few studies indicate a disorder in IL-17A, which can have a significant impact on the course of autoimmune diabetes [4]. The few available studies show an increased secretion of IL-17A from activated peripheral blood T cells in children with type 1 diabetes [5]. Recently, we have also reported that plasma IL-17A level is increased in type 2 diabetic patients [6]. These results suggest an involvement of the proinflammatory cytokine IL-17A in diabetes.

Diabetic retinopathy (DR), one of the most serious complications of diabetes, is a leading cause of blindness in working age people worldwide [7]. The pathogenesis of DR is complex and several vascular, inflammatory, and neuronal mechanisms are involved [8]. The involvement of inflammatory processes in inducing structural and functional alterations associated with DR is gaining increasing attention $[9,10]$. The inflammatory processes contribute to pathological changes in DR, including blood-retinal barrier (BRB) breakdown, retinal neovascularization, retinal glutamate metabolic dysfunction, and retinal neuronal apoptosis [11-14]. However, the evidence showing the inflammatory events in DR still stays in innate immunity driven by retinal macrophages and microglia. The role of IL-17A in DR has not yet been clarified.

Müller cells, the largest glial cells in the retina of vertebrates, cover practically the entire retinal thickness from the outer to the inner limiting membrane [15]. Müller glial cells are thought to play an essential role in maintaining the structural integrity of the retina and to participate in essential processes, such as glucose metabolism, neurotransmitter uptake, and retinal homeostasis $[16,17]$. Müller cell activation, as demonstrated by an upregulation of glial fibrillary acidic protein (GFAP), has been found in the retinas of both diabetic patients and diabetic rodents at early stages of DR [18-20]. Müller cell-derived vascular endothelial growth factor (VEGF) accumulation results in diabetic retinal neovascularization and vascular leakage [21]. Hyperglycemia-induced decreases in both glutamine synthetase (GS) and excitatory amino acid transporter (EAAT)1 in Müller cells may trigger apoptosis of retinal ganglion cells in DR, because of excitotoxicity caused by excessive glutamate [22, 23]. Accordingly, we hypothesized that Müller cells are a critical target or source of IL-17A in DR, by which IL-17A exacerbates BRB breakdown and retinal neuronal apoptosis, the major characteristics of DR.

Monoclonal antibodies (mAbs) recently emerged as new drug modalities to block soluble mediators of disease, such as cytokines and receptors [24]. Specific features, such as high specificity and target selectivity, offer advantages over conventional therapies with immunosuppressive and anti-inflammatory drugs used for the treatment of autoimmunity that may have side effects, such as opportunistic infections and toxicity [25]. Preclinical experiments in animal models as well as the current success of anti-IL-17A mAbs in clinical trials have validated the relevance of IL-17A as a target for the treatment of autoimmunity [25-29]. IL-17A signals through a heterodimeric receptor complex. The IL-17A, IL-17F, and IL-17A/F heterodimer ligands act through an IL-17RA/RC complex, and IL-17C acts through an IL-17RA/RE complex, with anti-IL-17RA antibodies able to block activities of all four ligand complexes [30]. In the present study, both anti-IL-17A-neutralizing mAb and anti-IL-17RA-neutralizing mAb were used in high glucose (HG)-treated rat Müller cell line (rMC-1) in vitro and streptozotocin (STZ)-induced DR mouse model in vivo to demonstrate an involvement of IL-17A in DR and a potential therapeutic strategy of the antibodies for DR.

\section{Materials and Methods}

\section{DR mouse model}

To induce diabetes, male 8-week-old C57BL/6 mice (Animal Core Facility of Nanjing Medical University, Nanjing, China) were treated for five consecutive days with intraperitoneal injections of STZ (Sigma-Aldrich, St. Louis, MO, USA) at a concentration of $50 \mathrm{mg} / \mathrm{kg}$ body weight, freshly made in $0.01 \mathrm{M}$ citrate buffer (pH 4.5). Vehicle injection ( $0.01 \mathrm{M}$ citrate buffer; $50 \mathrm{mg} / \mathrm{kg}$ body weight) was used as control. Two weeks after the first STZ injection, mice with blood glucose concentrations above $11.11 \mathrm{mM}$ were confirmed as successful diabetic models [31]. Mice were subjected to intravitreal injection or sacrificed three months after the onset 


\section{Cellular Physiology Cell Physiol Biochem 2017;41:960-972

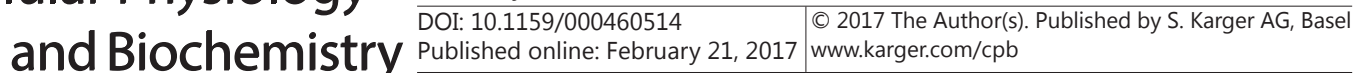 \\ Qiu et al.: Blocking IL-17A Alleviates DR}

of diabetes, according to this report that at 10-14 weeks after hyperglycemia of STZ mice, the retinas show representative DR pathology [32,33]. All animal procedures were in line with the Association for Research in Vision and Ophthalmology Statement for the Use of Animals in Ophthalmic and Vision Research, and were approved by the Institutional Animal Care and Use Committees of Nanjing Medical University.

\section{Intravitreal injection}

Intravitreal injections were performed on the mice anesthetized with isoflurane inhalation under a surgical microscope according to the description by Hombrebueno et al. [34] with some modifications. After a tiny incision was made by 30 -gauge needle $1 \mathrm{~mm}$ behind the limbus in deeply anesthetized mice, a 33-gauge needle mounted on a 10- $\mu$ l microsyringe (Hamilton, Reno, NE, USA) was inserted through the incision with a $45^{\circ}$ angle into the vitreous cavity. Anti-IL-17A-neutralizing $\mathrm{mAb}(2$ or $10 \mu \mathrm{g} / 2 \mu \mathrm{l}$ in PBS per eye) and antiIL-17RA-neutralizing mAb (0.4 or $2 \mu \mathrm{g} / 2 \mu \mathrm{l}$ in PBS per eye) (both from R\&D Systems, Minneapolis, MN, USA) were administered to the vitreous cavity of STZ mice, respectively. In the same method, $2 \mu$ of PBS was injected into the eye as control. Mice that were prepared for retinal angiography were sacrificed two weeks after intravitreal injection, while the rest were sacrificed two days after intravitreal injection.

\section{Cell culture and treatments}

The rMC-1 (Northwestern University, Chicago, IL, USA) was grown in Dulbecco's modified Eagle's medium (DMEM) supplemented with $5 \mathrm{mM}$ D-glucose. To mimic diabetes in vitro, rMC-1 was maintained in DMEM with high concentrations of D-glucose (15 or $25 \mathrm{mM}$ ) for $48 \mathrm{~h}$. Rat recombinant IL-17A $(10,25$ or $50 \mathrm{ng} / \mathrm{ml}$; BioVision, Milpitas, CA, USA), anti-IL-17A-neutralizing mAb (1 or $2 \mu \mathrm{g} / \mathrm{ml}$; R\&D Systems, Minneapolis, MN, USA), or/and anti-IL-17RA-neutralizing mAb (1 $\mu \mathrm{g} / \mathrm{ml}$; R\&D Systems, Minneapolis, MN, USA) were applied to rMC-1 with $25 \mathrm{mM}$ glucose DMEM for $24 \mathrm{~h}$.

\section{Western blot analysis}

Cells and retinas were isolated and homogenized in RIPA buffer with protease inhibitor cocktail (Roche Molecular Biochemicals, Indianapolis, IN, USA) according to the methods described previously [13]. Primary antibody against IL-17A (1:200) was from Santa Cruz Biotechnology (Dallas, Texas, USA); primary antibodies against IL-17RA (1:200), GFAP (1:10000), VEGF (1:1000), GS (1:1000), EAAT1 (1:1000), occludin (1:2000), zonula occludens-1 (ZO-1; 1:50), and $\beta$-actin (1:5000) were all from Abcam (Cambridge, MA, USA). Anti-rabbit or mouse IgG (1:5000, Rockland, Limerick, PA, USA) were used as secondary antibodies for detection. The images of bands were visualized and the intensities were quantified by Odyssey (LI-COR, Lincoln, NE, USA).

\section{Enzyme-linked immunosorbent assay (ELISA)}

Protein levels in retinal homogenates, cell culture media and cell homogenates were respectively determined using commercially available ELISA kits for IL-17A, GFAP and VEGF (Millpore, Temecula, CA or R\&D Systems, Minneapolis, MN, USA) on the basis of manufactures' instructions and the report of Huang et al. [13]. Protein titers were calculated by a reference to a standard curve constructed using known amounts of recombinant IL-17A, GFAP or VEGF.

\section{High performance liquid chromatography (HPLC)}

Previous to performing HPLC as described elsewhere [35], cells and retinas were homogenized in perchloric acid $(0.1 \mathrm{M})$ at $4^{\circ} \mathrm{C}$. The calculations of the glutamate peak area were carried out, and quantification was allowed by running standard amino acid solutions under the same conditions.

\section{Retinal leukostasis}

Leukocytes adherent to retinal vasculature were labeled with fluorescein isothiocyanate (FITC)conjugated concanavalin A (20 $\mu \mathrm{g} / \mathrm{ml}$ in PBS; Vector Laboratories, Burlingame, CA, USA), as previously described [36]. Briefly, after anesthetized with isoflurane inhalation, mice were perfused with $10 \mathrm{ml}$ of PBS from the left heart ventricle to remove erythrocytes and non-adherent leukocytes, followed by perfusion of FITC-concanavalin A ( $5 \mathrm{mg} / \mathrm{kg}$ body weight), and PBS of $10 \mathrm{ml}$ to flush out unbound concanavalin A. The eyes were enucleated and fixed in $4 \%$ paraformaldehyde (PFA) for $1 \mathrm{~h}$, and the retinas were flat mounted. Total number of adherent leukocytes per retina was counted under a fluorescence microscope. 


\section{Cellular Physiology Cell Physiol Biochem 2017;41:960-972

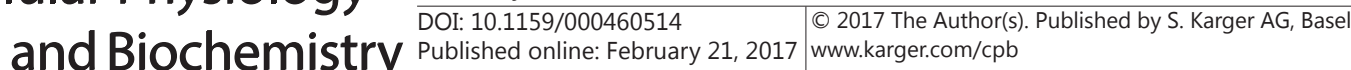 \\ Qiu et al.: Blocking IL-17A Alleviates DR}

\section{Retinal angiography}

Mice were anaesthetized with isoflurane inhalation and $1 \mathrm{ml}$ of PBS containing $50 \mathrm{mg} / \mathrm{ml} \mathrm{FITC-dextran}$ $\left(2 \times 10^{6}\right.$ molecular weight; Sigma-Aldrich, St. Louis, MO, USA $)$ was circulated through the left heart ventricle as described by Han et al. [37]. After perfused with FITC-dextran, mice were sacrificed, and enucleated eyecups were fixed in $4 \%$ PFA for $1 \mathrm{~h}$. The retinas were dissected, flat mounted onto glass slides, and viewed by fluorescence microscopy.

\section{Immunohistochemistry and TUNEL staining}

For rMC-1 immunocytochemistry, the cells grown on slides were fixed, permeabilized and blocked as previously reported [22]. Rabbit primary antibodies against GFAP (1:1000; Abcam, Cambridge, MA, USA), IL-17A (1:100; Santa Cruz Biotechnology, Dallas, Texas, USA) and IL-17RA (1:100; Abcam, Cambridge, MA, USA) were used to incubate the cells, respectively, overnight at $4^{\circ} \mathrm{C}$. After staining with anti-rabbit Alexa Fluor 647 (1:200; Jackson ImmunoResearch Laboratories, West Grove, PA, USA) for $2 \mathrm{~h}$ and Hoechst (1:1000; Dojindo Laboratories, Kumamoto, Japan) for $5 \mathrm{~min}$ at room temperature, the slides were observed by fluorescence microscopy. Average number of Hoechst-stained cells in three visual fields of each slide was counted as $100 \%$, and in the same three visual fields, average number of GFAP/Hoechst, IL-17A/Hoechst or IL-17RA/Hoechst double-positive cells in each slide was calculated as a percentage in the Hoechst-stained cells.

To prepare frozen retinal sections, the ocular anterior segments were removed and the remaining eyecups were fixed with 4\% PFA for $6 \mathrm{~h}$, followed by cryoprotected in a series of sucrose solution (10-30\%). The sectioned retinas were incubated with rabbit antibody against VEGF and mouse antibody against GS (both 1:200; Abcam, Cambridge, MA, USA) in sequence overnight at $4^{\circ} \mathrm{C}$. After the sections were stained with secondary antibodies including anti-rabbit Alexa Fluor 647 and anti-mouse FITC (1:200; Jackson ImmunoResearch Laboratories, West Grove, PA, USA) for $2 \mathrm{~h}$ at room temperature, DAPI (1:1000; Solarbio Science \& Technology, Beijing, China) staining for 5 min was used to label cell nucleus.

To detect apoptosis of retinal ganglion cells, the retinal sections that had been stained with NeuN (1:200; Abcam, Cambridge, MA, USA) were tested by TUNEL according to the manufacturer's protocol (Roche Diagnostics, Indianapolis, IN, USA). All the sections mentioned above were visualized by fluorescence microscopy and number of NeuN/TUNEL double-labeled cells was counted. In each section, three visual fields near retinal central area were counted as a sum, and an average of three sections in each retina was recorded as the number of NeuN/TUNEL double-labeled cells.

\section{Electron microscopy}

Isolated retinas were fixed in $2.5 \%$ glutaraldehyde and made into ultrathin sections as described previously [38]. The ultra-structure of the retinal ganglion cell layer (GCL) was observed under a transmission electron microscope (JEM-1230, JEOL, Tokyo, Japan).

\section{Statistical analysis}

The results are expressed as mean \pm standard deviation and statistical analyses were performed with Statistics Package for Social Science (SPSS, 12.0). A Student's $t$ test was used for comparisons between two groups, while group differences were evaluated by one-way analysis of variance (ANOVA), followed by post hoc analysis. Results were considered statistically significant at $P<0.05$.

\section{Results}

Expression of IL-17A and IL-17RA is upregulated in both STZ-induced diabetic retina and $H G$-treated $r M C-1$

To confirm that STZ induced diabetic changes, we measured blood glucose concentration and body weight at two weeks after first STZ injection. Compared with vehicle-treated control mice, STZ-treated mice had an increased blood glucose concentration $(18.73 \pm 2.35 \mathrm{mM}, n=$ 45 , vs. $8.35 \pm 0.98 \mathrm{mM}, n=24, P<0.01)$ and a decreased body weight $(20.56 \pm 1.00 \mathrm{~g}, n=45$, vs. $23.81 \pm 0.97 \mathrm{~g}, n=24, P<0.01$ ). At three months after the onset of diabetes, STZ-treated mice manifested an upregulated IL-17A and IL-17RA expression as well as an increased IL17A content in the retina, with respect to vehicle-treated control mice (Fig. 1A and 1B). 


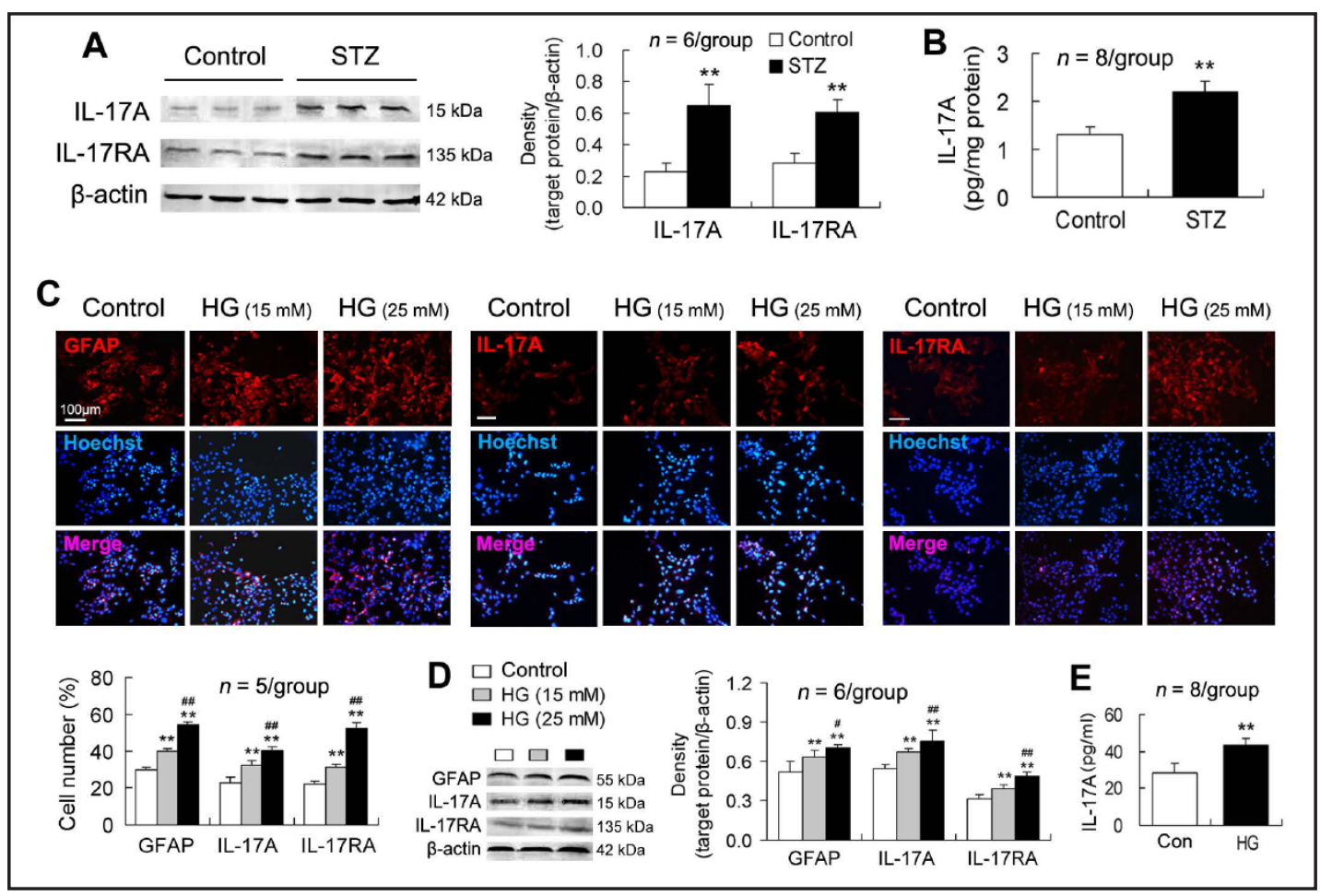

Fig. 1. IL-17A and IL-17RA expression is upregulated in both STZ-induced diabetic retina and HG-treated rMC-1. (A) Retinal expression levels of IL-17A and IL-17RA tested by Western blot. (B) IL-17A content in the retina measured by ELISA. (C) Immunofluorescent staining on rMC-1 cultures indicates the double-labeled cells of GFAP/Hoechst, IL-17A/Hoechst and IL-17RA/Hoechst. Percentages of the double-labeled cells in the histogram were calculated as described in Materials and Methods. (D) Protein expression levels in rMC1 tested by Western blot. (E) IL-17A concentration in cultured rMC-1 supernatants measured by ELISA. ${ }^{* *} P$ $<0.01$ vs. control; $\# P<0.05$, \#\#P<0.01, vs. HG $(15 \mathrm{mM})$.

In HG-treated rMC-1, the numbers of both IL-17A- and IL-17RA-stained cells were increased (Fig. 1C), as well as the expression of both IL-17A and IL-17RA was upregulated (Fig. 1D). These effects of $25 \mathrm{mM} \mathrm{HG}$ were larger than those of $15 \mathrm{mM}$ HG (Fig. 1C and 1D). Moreover, IL-17A concentration in cultured rMC-1 supernatants was increased in HG condition (Fig. 1E). In addition, both GFAP-labeled cell number and GFAP expression level were increased in HG-treated rMC-1 relative to in control rMC-1 (Fig. 1C and 1D). This confirms an activation of rMC-1 by HG.

\section{IL-17A exacerbates $H G$-induced $r M C$-1 activation and dysfunction via IL-17RA}

GFAP and VEGF expression was upregulated, but GS and EAAT1 expression was downregulated in rMC-1, when the cells were exposed to HG (25 mM) (Fig. 2A). Importantly, treatment of rMC-1 with IL-17A (10, 25 or $50 \mathrm{ng} / \mathrm{ml})$ exacerbated the HG-induced changes in expression of GFAP, VEGF, GS and EAAT1 (Fig. 2A). These effects of IL-17A were larger at the higher concentration than at the lower concentration. In contrast, anti-IL-17A-neutralizing $\mathrm{mAb}(1$ or $2 \mu \mathrm{g} / \mathrm{ml})$ reduced the HG-induced changes in expression of GFAP, VEGF, GS and EAAT1 in rMC-1 (Fig. 2A). In addition, anti-IL-17RA-neutralizing mAb $(1 \mu \mathrm{g} / \mathrm{ml})$ not only reduced the HG-induced changes, but also blocked the IL-17A effects (Fig. 2A). Similarly, GFAP content in the cultured rMC-1 lysates, VEGF concentration in the cultured rMC-1 supernatants, and glutamate content in the rMC-1 lysates were increased in HG condition and were further increased by IL-17A exposure (Fig. 2B, 2C and 2D). Either anti-IL-17A mAb or anti-IL-17RA mAb reduced the HG-induced changes in production or secretion of GFAP, VEGF and glutamate in rMC-1 (Fig. 2B, 2C and 2D). Anti-IL-17RA mAb also blocked the IL17A effects (Fig. 2B, 2C and 2D).

\section{KARGER}




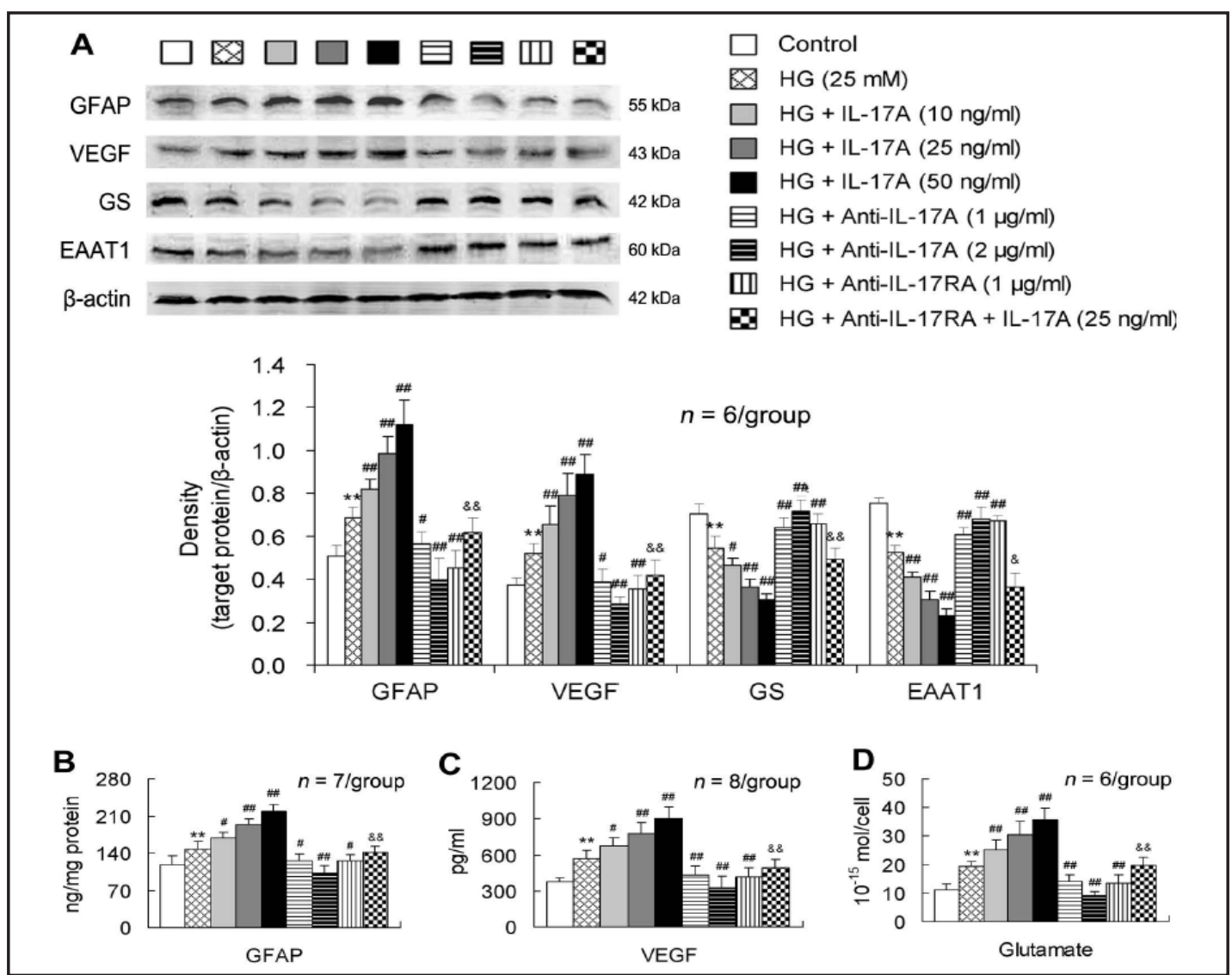

Fig. 2. IL-17A exacerbates HG-induced rMC-1 activation and dysfunction via IL-17RA. (A) Expression changes of the proteins related to Müller cell activation (GFAP) and function (VEGF, GS and EAAT1) in rMC-1. (B) GFAP content in rMC-1 homogenates tested by ELISA. (C) VEGF concentration in rMC-1 culture media tested by ELISA. (D) Glutamate content in rMC-1 homogenates measured by HPLC. The cutline at the upper right is shared by (A-D). ${ }^{* *} P<0.01$ vs. control; $\# P<0.05$, \#\#P<0.01, vs. HG $(25 \mathrm{mM}) ; \& P<0.05, \& \& P<0.01$, vs. $\mathrm{HG}+\mathrm{IL}-17 \mathrm{~A}(25 \mathrm{ng} / \mathrm{ml})$.

Either anti-IL-17A mAb or anti-IL-17RA mAb alleviate retinal Müller cell activation and dysfunction in STZ-induced DR

GFAP and VEGF expression and glutamate content were increased, whereas GS and EAAT1 expression was downregulated in the retina of STZ-induced DR (Fig. 3A and 3B). Notably, intravitreal injections of anti-IL-17A mAb $(2$ or $10 \mu \mathrm{g} / 2 \mu \mathrm{l})$ or anti-IL-17RA mAb $(0.4$ or $2 \mu \mathrm{g} / 2 \mu \mathrm{l})$ in STZ-treated mice ameliorated the retinal changes in expression or production of GFAP, VEGF, GS, EAAT1 and glutamate, compared with intravitreal injection of PBS (Fig. 3A and 3B). As a control of intravitreal administration, PBS intravitreal injection in STZ-treated mice did not alter the diabetes-induced retinal disorders (Fig. 3A and 3B).

To demonstrate that the retinal disorders were linked to Müller cell dysfunction, we examined the change in co-expression of GS, a marker of retinal Müller cells, and VEGF in the retina using fluorescent immunohistochemistry. As expected, GS expression was diminished whereas VEGF expression was increased in STZ-induced diabetic retina (Fig. 3C). Importantly, the co-expression of GS and VEGF was enhanced in diabetic retina, and this enhancement was reduced by intravitreal treatments with anti-IL-17A mAb or anti-IL-17RA mAb (Fig. 3C).

Both anti-IL-17A $m A b$ and anti-IL-17RA $m A b$ ameliorate BRB breakdown in STZ-induced $D R$

BRB breakdown was determined by retinal vascular leukostasis, vascular leakage, and expression of occludin and $\mathrm{ZO}-1$, the interendothelial tight junction proteins. The number 


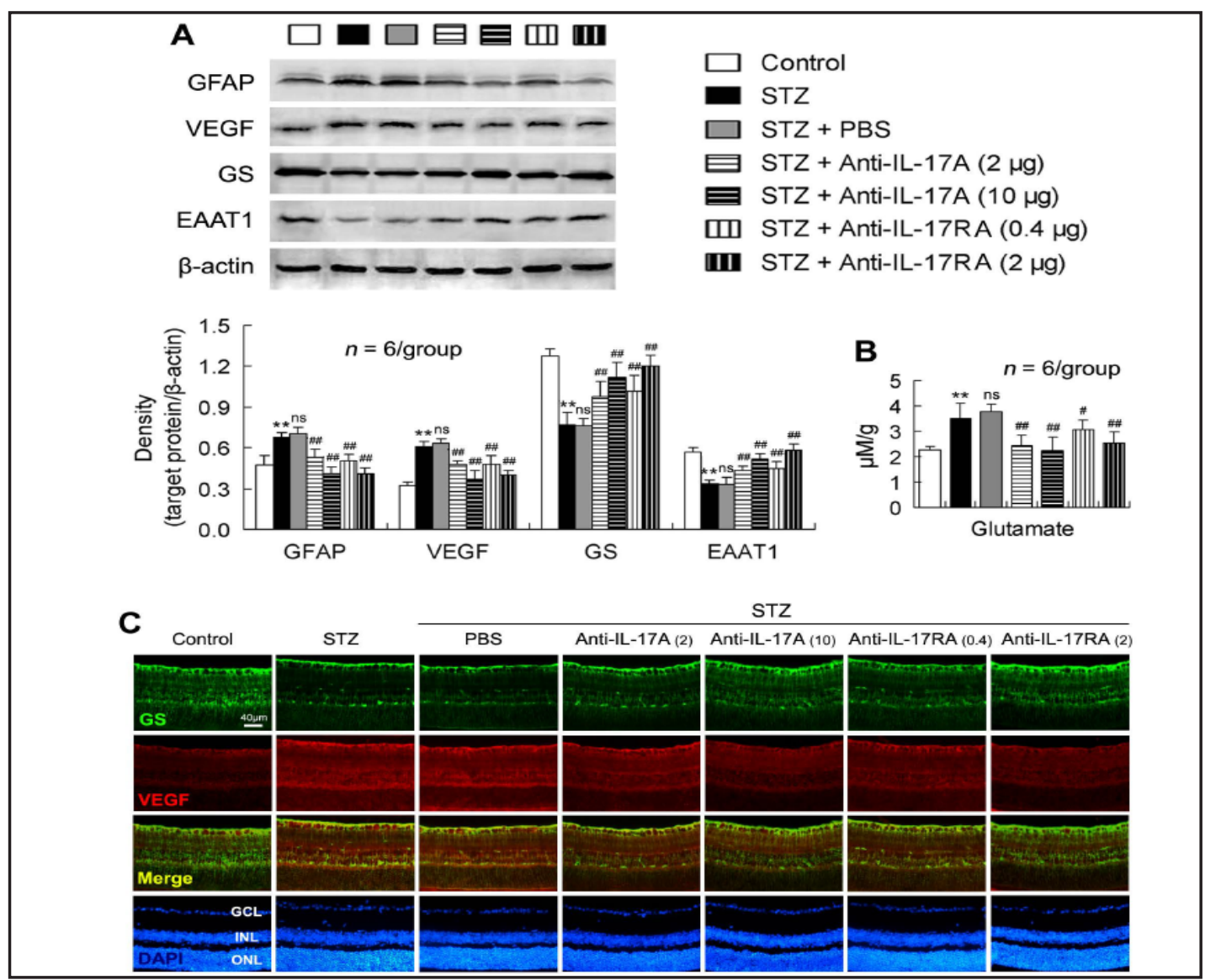

Fig. 3. Either anti-IL-17A-neutralizing mAb or anti-IL-17RA-neutralizing mAb alleviate retinal Müller cell activation and dysfunction in STZ-induced DR. At three months after the onset of diabetes, the mice were injected with anti-IL-17A mAb or anti-IL-17RA mAb in the vitreous cavity and two days later, the protein expression $(A)$ and glutamate content $(B)$ in the retina were determined. The cutline at the upper right is shared by $(A$ and $B) .{ }^{* *} P<0.01$ vs. control; $\# P<0.05$, \#\#P<0.01, vs. STZ + PBS; ns: no significance vs. STZ. (C) Immunofluorescent staining on retinal cross sections indicates a co-expression of GS that labels Müller cells and VEGF. GCL: ganglion cell layer; INL: inner nuclear layer; ONL: outer nuclear layer.

of vascular adherent leukocytes was increased in the retina of STZ-induced DR (Fig. 4A). Intravitreal administration with either anti-IL-17A mAb $(2$ or $10 \mu \mathrm{g} / 2 \mu \mathrm{l})$ or anti-IL-17RA $\mathrm{mAb}(0.4$ or $2 \mu \mathrm{g} / 2 \mu \mathrm{l})$ in diabetic mice decreased the number of retinal vascular adherent leukocytes compared with PBS intravitreal administration (Fig. 4A). This effect of both mAbs was stronger at a higher dose than at a lower dose.

Expression of both occludin and ZO-1 was downregulated in the retina of STZ-induced DR (Fig. 4B). Intravitreal treatments with anti-IL-17A mAb or anti-IL-17RA mAb reduced the downregulated expression of occludin and ZO-1 in the retina of DR compared with PBS intravitreal treatment (Fig. 4B).

FITC-labeled dextran leakage out of the retinal vessels was enhanced in diabetic mice relative to in control mice (Fig. 4C). Either intravitreal treatment with anti-IL-17A mAb or anti-IL-17RA mAb alleviated the retinal vascular leakage in DR compared with intravitreal treatment with PBS (Fig. 4C).

Anti-IL-17A $m A b$ or anti-IL-17RA $m A b$ reduce retinal ganglion cell apoptosis in STZinduced $D R$

Number of NeuN/TUNEL double-positive cells in the GCL was more in STZ-induced diabetic mice than in control mice (Fig. 5A). This increased NeuN/TUNEL double-positive cell 


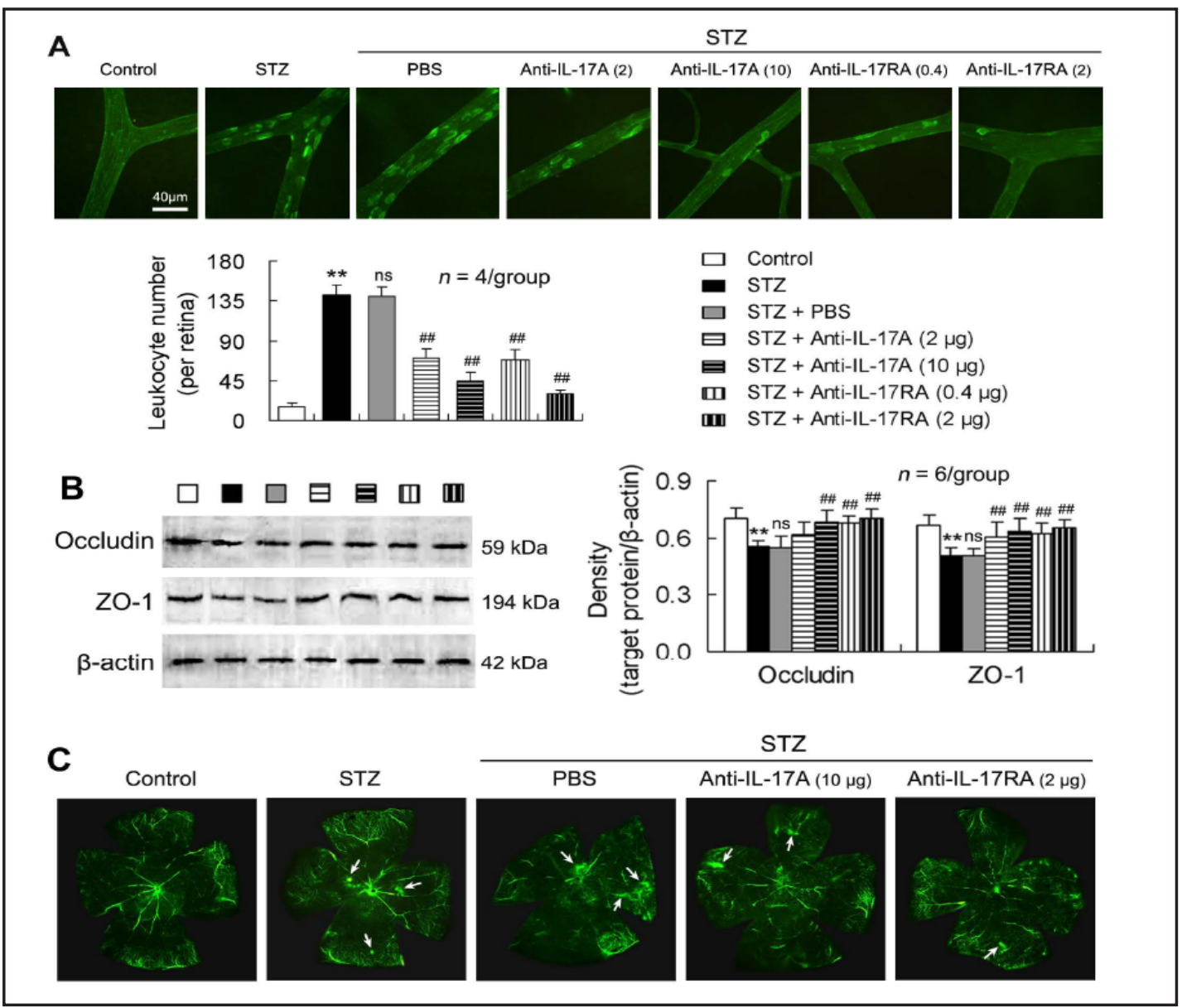

Fig. 4. Anti-IL-17A mAb or anti-IL-17RA mAb ameliorate BRB breakdown in STZ-induced DR. At three months after the onset of diabetes, the mice were injected with anti-IL-17A mAb or anti-IL-17RA mAb in the vitreous cavity and two days later, the adherent leukocytes that were labeled by FITC-concanavalin A within the retinal vessels were observed and counted in each retina (A), and expression levels of the tight junction proteins were determined (B). The cutline at the middle right is shared by (A and $\mathrm{B}$ ). ${ }^{* *} P<0.01$ vs. control; $\# \# P<0.01$, vs. STZ + PBS; ns: no significance vs. STZ. (C) Angiographic images of flat-mounted retinas show retinal vascular leakage. Retinal angiography was performed two weeks after intravitreal injection. The arrows indicate vascular FITC-dextran exudation.

number in diabetic retina was reduced by intravitreal administration with anti-IL-17A mAb or anti-IL-17RA mAb (Fig. 5A). Further, electron microscopy displayed the morphological characteristics of apoptosis of retinal ganglion cells in DR, with chromatin margination, chromatin condensation, vacuolation, and cell deeply stained and shrunken (Fig. 5B). The morphological changes of retinal neuronal apoptosis in DR were ameliorated by either treatment with anti-IL-17A mAb or anti-IL-17RA mAb in the vitreous cavity (Fig. 5B).

\section{Discussion}

IL-17A expression and production as well as IL-17RA expression were increased in the retina of DR, suggesting that the proinflammatory cytokine IL-17A is involved in DR process. Further, HG induced retinal Müller cell expression and secretion of IL-17A and expression of IL-17RA. These findings suggest that retinal Müller cells are a source and target for IL-17A in DR. IL-17A is generally thought as a specific cytokine of Th17 cells. Since the retina does 


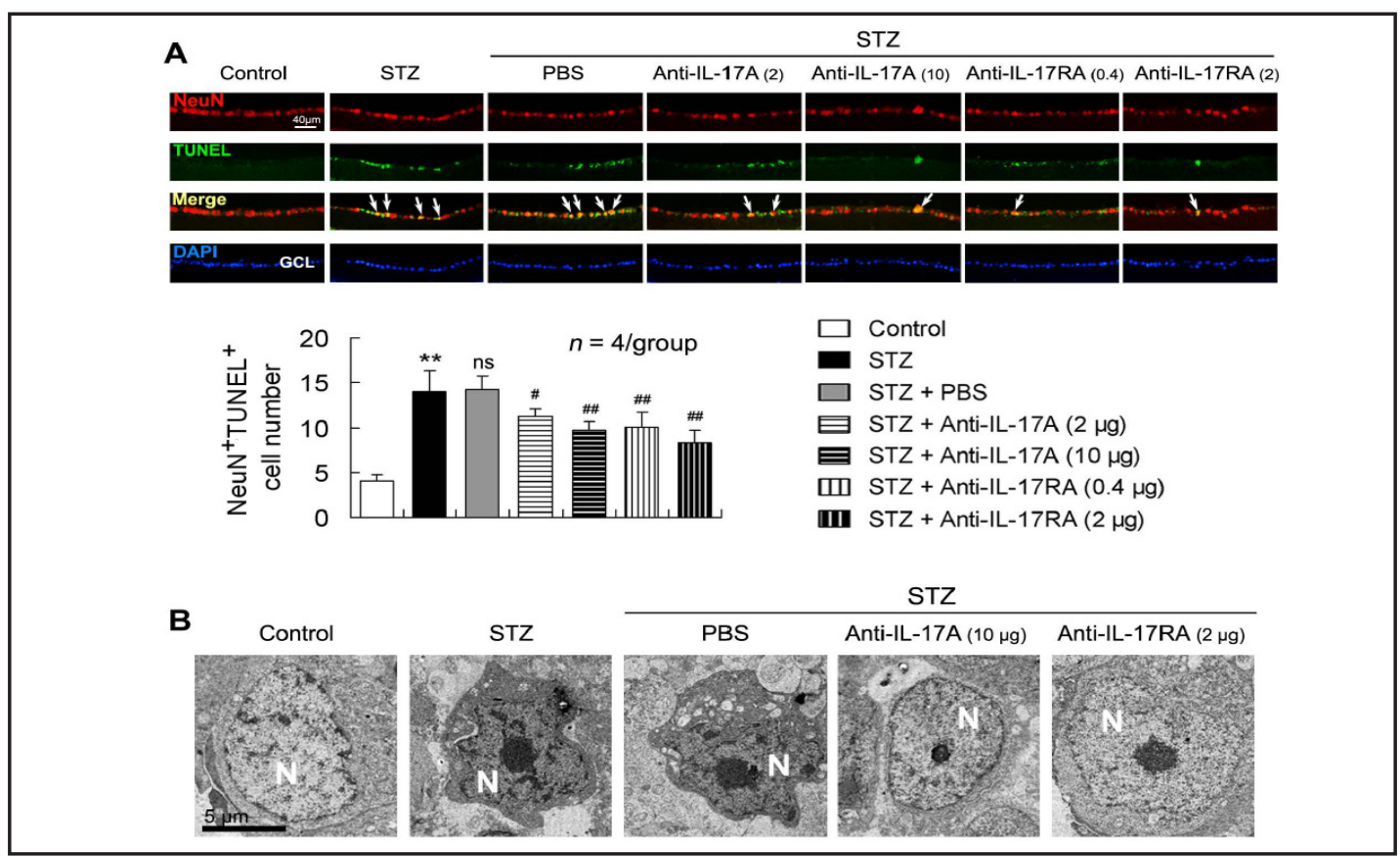

Fig. 5. Both anti-IL-17A and anti-IL-17RA mAbs reduce retinal ganglion cell apoptosis in DR. (A) Immunofluorescent staining on retinal cross sections displays ganglion cell apoptosis. The NeuN/TUNEL double-positive cells in the GCL, indicated by the arrows, represent apoptotic ganglion cells. The double-positive cell number is expressed as described in Materials and Methods. ${ }^{* *} P<0.01$ vs. control; $\# P<0.05$, \#\#P<0.01, vs. STZ + PBS; ns: no significance vs. STZ. (B) Electron microscopic images show ultrastructural changes of apoptosis of retinal ganglion cells. The most striking ultrastructural feature of apoptosis is in the nucleus (N). Control exhibits a normal ganglion cell morphology. Apoptotic retinal ganglion cells (STZ or STZ plus PBS treatments) were characterized by a prominent increase in electron density throughout the entire nuclear matrix, nuclear membrane folds and collapse, and chromatin condensation. The cell cytoplasm became more highly concentrated and had vacuolation.

not have well-developed lymphatic drainage [39], the ocular tissue is often deemed to be immunoprivileged. Recently, a few studies suggest that Th17 cells infiltrate from circulation into the eye across retinal vascular endothelium to participate in inflammatory response during degeneration of retinal pigment epithelium (RPE) [40] and in autoimmune uveitis [41]. Here we for the first time show that IL-17A is also produced by retinal Müller cells in DR.

Retinal Müller cells play a critical role in DR occurrence and development. Activation of Müller cells, shown by an increased expression of GFAP, is a sign of human early DR pathology [42]. Müller cells are also a major source of retinal VEGF in diabetic mice and Müller cell-derived VEGF plays an essential and causative role in retinal inflammation and vascular leakage in DR [21]. In addition, retinal glutamate accumulation, because of Müller cell downregulation of GS and EAAT1 in DR, leads to excitotoxicity particularly to retinal neurons $[43,44]$. These disorders in expression, production or/and secretion of GFAP, VEGF, GS, EAAT1 and glutamate in DR were demonstrated in the current study. Importantly, exogenously-added IL-17A exacerbated HG-induced rMC-1 activation and functional impairment. These findings suggest that IL-17A induces retinal Müller cell inflammatory response, by which DR occurs and develops.

BRB deterioration, which is characterized by reduced blood flow, leukostasis, increased vascular permeability, and pathological angiogenesis, is a cardinal manifestation of DR [45, 46]. As expected, diabetic retina had an increased leukostasis and vascular leakage as well as a downregulated expression of the tight junction proteins occludin and ZO-1. BRB is composed of both an inner and an outer barrier. The outer BRB is formed by RPE cell layer and the 
inner BRB comprises the microvascular endothelium that lines the retinal microvasculature [47]. The tight junctions located between these cells mediate highly selective diffusion of molecules from the blood to the retina. Our present results showing a downregulated retinal expression of the tight junction proteins in DR represent a mechanism underlying BRB breakdown. As a support, other authors present that IL-17A compromises ARPE-19 (RPE cell line) monolayer barrier function in association with a disrupted distribution of the junction proteins occludin and ZO-1 [48]. In addition to BRB deterioration, retinal neuronal apoptosis is reported in DR patients and mice $[49,50]$. In retinal neurons, ganglion cells are the best studied neurons with respect to the effect of diabetes [51, 52]. In this study, the increased retinal ganglion cell apoptosis in DR was identified. We explain that the decrease in glutamate recycling in dysfunctional Müller cells in response to IL-17A is a critical cause for neuronal death in DR.

Antibodies targeting IL-17A or its receptor, IL-17RA, are approved to treat psoriasis and are being evaluated for other autoimmune conditions [53,54]. We found that intravitreal treatment with anti-IL-17A-neutralizing $\mathrm{mAb}$ alleviated DR-like pathology, including retinal Müller cell activation and dysfunction, BRB breakdown, and retinal ganglion cell apoptosis. The effective remedy of DR by anti-IL-17A mAb demonstrates that IL-17A, which may be produced by and secreted from retinal Müller cells in DR, participates in retinal inflammatory events and thereby promotes DR processes. IL-17A also probably originates from Th17 cells that may infiltrate into the retinal parenchyma from the blood vessels through the damaged BRB in DR. Further evidence is needed to identify this hypothesis. An anti-IL-17A mAb that exhibits high affinity and protective in vivo capacity even in germline configuration has implications for B-cell tolerance as well as the regulation of cytokine networks [24]. Here we provide new evidence showing that anti-IL-17A mAb treatment via the vitreous cavity is a promising therapeutic strategy for DR.

In addition to anti-IL-17A-neutralizing $\mathrm{mAb}$, anti-IL-17RA-neutralizing $\mathrm{mAb}$ also ameliorated the DR-like pathology. Moreover, IL-17A effect of aggravating rMC-1 activation and functional impairment was blocked by anti-IL-17RA mAb. The results demonstrate that IL-17RA mediates IL-17A action in DR. Indeed, IL-17RA was expressed by rMC-1 in this study. Although IL-17RA is also expressed by other retinal cells, such as retinal astrocytes and RPE, in vitro investigation shows a weak inflammatory effect of RPE cells in response to IL-17A [55]. Accordingly, we propose that retinal Müller cells are a predominant target for IL-17A in DR. Müller cell abnormality has been linked to BRB breakdown and retinal neuronal dysfunction and death [56]. This supports our hypothesis that by inflammatory injury to retinal Müller cells, IL-17A accelerates DR progression. Thus, blocking IL-17RA on retinal Müller cells alleviates DR-like pathology.

Within the past decade, anti-VEGF agents have revolutionized the treatment of several retinal diseases, including diabetic macular edema (DME), for which anti-VEGF agents are now accepted as first-line treatment [56]. In contrast, the effects of anti-VEGF agents on the severity of DR aside from DME are more incompletely established [56]. Therefore, antiVEGF agents are being explored as an alternative and adjunctive therapeutic strategy in the treatment of DR [57-59]. In the current study, IL-17A promoted VEGF secretion from retinal Müller cells, which was involved in DR inflammatory pathogenesis. Accordingly, a combined treatment with anti-VEGF and anti-IL-17A or anti-IL-17RA may be more promising for blocking VEGF and ameliorating DR than a single treatment. This hypothesis needs to be further investigated particularly in human Müller cells such as the human Müller cell line MIO-M1.

In summary, both IL-17A and IL-17RA production is increased in retinal tissue and rMC-1 in DR. Exogenously-added IL-17A exacerbates HG-induced rMC-1 activation and dysfunction, whereas either anti-IL-17A mAb or anti-IL-17RA mAb alleviate the HG-induced rMC-1 disorders. Blocking IL-17A via intravitreal injections of anti-IL-17A mAb or anti-IL17RA mAb effectively ameliorates DR-like pathology, including retinal Müller cell disorders, BRB disruption, and retinal ganglion cell apoptosis. These findings establish that IL-17A participates in inflammatory events of DR and promotes DR pathology at least in part by 


\section{Cellular Physiology Cell Physiol Biochem 2017;41:960-972

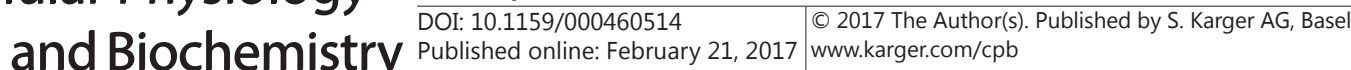 \\ Qiu et al.: Blocking IL-17A Alleviates DR}

affecting retinal Müller cells, and suggest that a novel therapeutic option blocking IL-17A or IL-17RA is beneficial for slowing down DR processes.

\section{Acknowledgments}

This study was supported by the National Basic Research Program of China (973 Program, No. 2013CB967500 and No. 2011CB510200), the National Natural Science Foundation of China (No. 81170855), Jiangsu Provincial Special Program of Medical Science (BL2014089), and Six Talent Peak Project in Jiangsu Province (2013-WSW-015).

\section{Disclosure Statement}

The authors declare that there is no conflict of interest regarding the publication of this paper.

\section{References}

1 Gaffen SL: Recent advances in the IL-17 cytokine family. Curr Opin Immunol 2011;23:613-619.

2 Matusevicius D, Kivisakk P, He B, Kostulas N, Ozenci V, Fredrikson S, Link H: Interleukin-17 mRNA expression in blood and CSF mononuclear cells is augmented in multiple sclerosis. Mult Scler 1999;5:101104.

3 Teunissen MB, Koomen CW, de Waal Malefyt R, Wierenga EA, Bos JD: Interleukin-17 and interferon-gamma synergize in the enhancement of proinflammatory cytokine production by human keratinocytes. J Invest Dermatol 1998;111:645-649.

4 Semeran K, Pawlowski P, Lisowski L, Szczepaniak I, Wojtowicz J, Lawicki S, Bakunowicz-Lazarczyk A, Bossowski A: Plasma levels of IL-17, VEGF, and adrenomedullin and S-cone dysfunction of the retina in children and adolescents without signs of retinopathy and with varied duration of diabetes. Mediators Inflamm 2013;2013:274726.

5 Marwaha AK, Crome SQ, Panagiotopoulos C, Berg KB, Qin H, Ouyang Q, Xu L, Priatel JJ, Levings MK, Tan R: Cutting edge: Increased IL-17-secreting T cells in children with new-onset type 1 diabetes. J Immunol 2010;185:3814-3818.

6 Hang H, Yuan S, Yang Q Yuan D, Liu Q: Multiplex bead array assay of plasma cytokines in type 2 diabetes mellitus with diabetic retinopathy. Mol Vis 2014;20:1137-1145.

7 Cheung N, Mitchell P, Wong TY: Diabetic retinopathy. Lancet 2010;376:124-136.

8 Semeraro F, Cancarini A, dell'Omo R, Rezzola S, Romano MR, Costagliola C: Diabetic Retinopathy: Vascular and Inflammatory Disease. J Diabetes Res 2015;2015:582060.

-9 Gariano RF, Gardner TW: Retinal angiogenesis in development and disease. Nature 2005;438:960-966.

$>10$ Wang LL, Chen H, Huang K, Zheng L: Elevated histone acetylations in Muller cells contribute to inflammation: a novel inhibitory effect of minocycline. Glia 2012;60:1896-1905.

11 Adamis AP, Berman AJ: Immunological mechanisms in the pathogenesis of diabetic retinopathy. Semin Immunopathol 2008;30:65-84.

12 Hazra S, Rasheed A, Bhatwadekar A, Wang X, Shaw LC, Patel M, Caballero S, Magomedova L, Solis N, Yan Y, Wang W, Thinschmidt JS, Verma A, Li Q, Levi M, Cummins CL, Grant MB: Liver X receptor modulates diabetic retinopathy outcome in a mouse model of streptozotocin-induced diabetes. Diabetes 2012;61:3270-3279.

13 Huang H, Gandhi JK, Zhong X, Wei Y, Gong J, Duh EJ, Vinores SA: TNFalpha is required for late BRB breakdown in diabetic retinopathy, and its inhibition prevents leukostasis and protects vessels and neurons from apoptosis. Invest Ophthalmol Vis Sci 2011;52:1336-1344.

14 Shen X, Xie B, Cheng Y, Jiao Q, Zhong Y: Effect of pigment epithelium derived factor on the expression of glutamine synthetase in early phase of experimental diabetic retinopathy. Ocul Immunol Inflamm 2011;19:246-254.

15 Fernandez-Sanchez L, Lax P, Campello L, Pinilla I, Cuenca N: Astrocytes and Muller Cell Alterations During Retinal Degeneration in a Transgenic Rat Model of Retinitis Pigmentosa. Front Cell Neurosci 2015;9:484.

16 Chong RS, Martin KR: Glial cell interactions and glaucoma. Curr Opin Ophthalmol 2015;26:73-77. 


\section{Cellular Physiology Cell Physiol Biochem 2017;41:960-972

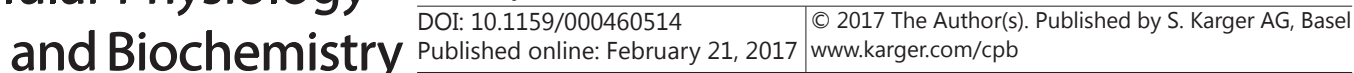 \\ Qiu et al.: Blocking IL-17A Alleviates DR}

17 Reichenbach A, Bringmann A: New functions of Muller cells. Glia 2013;61:651-678.

18 Barber AJ, Antonetti DA, Gardner TW: Altered expression of retinal occludin and glial fibrillary acidic protein in experimental diabetes. The Penn State Retina Research Group. Invest Ophthalmol Vis Sci 2000;41:3561-3568.

19 Carrasco E, Hernandez C, Miralles A, Huguet P, Farres J, Simo R: Lower somatostatin expression is an early event in diabetic retinopathy and is associated with retinal neurodegeneration. Diabetes Care 2007;30:2902-2908.

20 Garcia-Ramirez M, Hernandez C, Villarroel M, Canals F, Alonso MA, Fortuny R, Masmiquel L, Navarro A, Garcia-Arumi J, Simo R: Interphotoreceptor retinoid-binding protein (IRBP) is downregulated at early stages of diabetic retinopathy. Diabetologia 2009;52:2633-2641.

-21 Wang J, Xu X, Elliott MH, Zhu M, Le YZ: Muller cell-derived VEGF is essential for diabetes-induced retinal inflammation and vascular leakage. Diabetes 2010;59:2297-2305.

22 Gu L, Xu H, Wang F, Xu G, Sinha D, Wang J, Xu JY, Tian H, Gao F, Li W, Lu L, Zhang J, Xu GT: Erythropoietin exerts a neuroprotective function against glutamate neurotoxicity in experimental diabetic retina. Invest Ophthalmol Vis Sci 2014;55:8208-8222.

23 Zeng K, Xu H, Chen K, Zhu J, Zhou Y, Zhang Q, Mantian M: Effects of taurine on glutamate uptake and degradation in Muller cells under diabetic conditions via antioxidant mechanism. Mol Cell Neurosci 2010;45:192-199.

-24 Dallenbach K, Maurer P, Rohn T, Zabel F, Kopf M, Bachmann MF: Protective effect of a germline, IL17-neutralizing antibody in murine models of autoimmune inflammatory disease. Eur J Immunol 2015;45:1238-1247.

25 Bach JF: Immunosuppressive therapy of autoimmune diseases. Immunol Today 1993;14:322-326.

26 Nakae S, Nambu A, Sudo K, Iwakura Y: Suppression of immune induction of collagen-induced arthritis in IL-17-deficient mice. J Immunol 2003;171:6173-6177.

27 Komiyama Y, Nakae S, Matsuki T, Nambu A, Ishigame H, Kakuta S, Sudo K, Iwakura Y: IL-17 plays an important role in the development of experimental autoimmune encephalomyelitis. J Immunol 2006;177:566-573.

28 Uyttenhove C, Van Snick J: Development of an anti-IL-17A auto-vaccine that prevents experimental autoimmune encephalomyelitis. Eur J Immunol 2006;36:2868-2874.

29 Rohn TA, Jennings GT, Hernandez M, Grest P, Beck M, Zou Y, Kopf M, Bachmann MF: Vaccination against IL17 suppresses autoimmune arthritis and encephalomyelitis. Eur J Immunol 2006;36:2857-2867.

-30 Ramirez-Carrozzi V, Sambandam A, Luis E, Lin Z, Jeet S, Lesch J, Hackney J, Kim J, Zhou M, Lai J, Modrusan Z, Sai T, Lee W, Xu M, Caplazi P, Diehl L, de Voss J, Balazs M, Gonzalez L, Jr., Singh H, Ouyang W, Pappu R: IL-17C regulates the innate immune function of epithelial cells in an autocrine manner. Nat Immunol 2011;12:1159-1166.

31 Caporali A, Meloni M, Nailor A, Mitic T, Shantikumar S, Riu F, Sala-Newby GB, Rose L, Besnier M, Katare R, Voellenkle C, Verkade P, Martelli F, Madeddu P, Emanueli C: p75(NTR)-dependent activation of NF-kappaB regulates microRNA-503 transcription and pericyte-endothelial crosstalk in diabetes after limb ischaemia. Nat Commun 2015;6:8024.

32 Lai AK, Lo AC: Animal models of diabetic retinopathy: summary and comparison. J Diabetes Res 2013;2013:106594.

-33 Robinson R, Barathi VA, Chaurasia SS, Wong TY, Kern TS: Update on animal models of diabetic retinopathy: from molecular approaches to mice and higher mammals. Dis Model Mech 2012;5:444-456.

-34 Hombrebueno JR, Luo C, Guo L, Chen M, Xu H: Intravitreal Injection of Normal Saline Induces Retinal Degeneration in the C57BL/6J Mouse. Transl Vis Sci Technol 2014;3:3.

-35 Zhang Y, Zhang J, Wang Q, Lei X, Chu Q, Xu GT, Ye W: Intravitreal injection of exendin-4 analogue protects retinal cells in early diabetic rats. Invest Ophthalmol Vis Sci 2011;52:278-285.

-36 Joussen AM, Murata T, Tsujikawa A, Kirchhof B, Bursell SE, Adamis AP: Leukocyte-mediated endothelial cell injury and death in the diabetic retina. Am J Pathol 2001;158:147-152.

-37 Han Z, Guo J, Conley SM, Naash MI: Retinal angiogenesis in the Ins2(Akita) mouse model of diabetic retinopathy. Invest Ophthalmol Vis Sci 2013;54:574-584.

-38 Li D, Yang F, Cheng H, Liu C, Sun M, Wu K, Ai M: Protective effects of total flavonoids from Flos Puerariae on retinal neuronal damage in diabetic mice. Mol Vis 2013;19:1999-2010. 


\section{Cellular Physiology Cell Physiol Biochem 2017;41:960-972

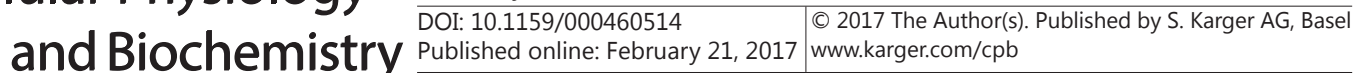 \\ Qiu et al.: Blocking IL-17A Alleviates DR}

-39 Schroedl F, Brehmer A, Neuhuber WL, Kruse FE, May CA, Cursiefen C: The normal human choroid is endowed with a significant number of lymphatic vessel endothelial hyaluronate receptor 1 (LYVE-1)positive macrophages. Invest Ophthalmol Vis Sci 2008;49:5222-5229.

40 Zhao Z, Xu P, Jie Z, Zuo Y, Yu B, Soong L, Sun J, Chen Y, Cai J: gammadelta T cells as a major source of IL-17 production during age-dependent RPE degeneration. Invest Ophthalmol Vis Sci 2014;55:6580-6589.

41 Bharadwaj AS, Schewitz-Bowers LP, Wei L, Lee RW, Smith JR: Intercellular adhesion molecule 1 mediates migration of Th1 and Th17 cells across human retinal vascular endothelium. Invest Ophthalmol Vis Sci 2013;54:6917-6925.

-42 Vujosevic S, Micera A, Bini S, Berton M, Esposito G, Midena E: Aqueous Humor Biomarkers of Muller Cell Activation in Diabetic Eyes. Invest Ophthalmol Vis Sci 2015;56:3913-3918.

-43 Hernandez C, Bogdanov P, Corraliza L, Garcia-Ramirez M, Sola-Adell C, Arranz JA, Arroba AI, Valverde AM, Simo R: Topical Administration of GLP-1 Receptor Agonists Prevents Retinal Neurodegeneration in Experimental Diabetes. Diabetes 2016;65:172-187.

44 Jindal V: Neurodegeneration as a primary change and role of neuroprotection in diabetic retinopathy. Mol Neurobiol 2015;51:878-884.

45 Cerani A, Tetreault N, Menard C, Lapalme E, Patel C, Sitaras N, Beaudoin F, Leboeuf D, De Guire V, Binet F, Dejda A, Rezende FA, Miloudi K, Sapieha P: Neuron-derived semaphorin 3A is an early inducer of vascular permeability in diabetic retinopathy via neuropilin-1. Cell Metab 2013;18:505-518.

-46 Tang J, Kern TS: Inflammation in diabetic retinopathy. Prog Retin Eye Res 2011;30:343-358.

47 Campbell M, Humphries P: The blood-retina barrier: tight junctions and barrier modulation. Adv Exp Med Biol 2012;763:70-84.

48 Chen Y, Yang P, Li F, Kijlstra A: The effects of Th17 cytokines on the inflammatory mediator production and barrier function of ARPE-19 cells. PLoS One 2011;6:e18139.

49 Mohr S, Xi X, Tang J, Kern TS: Caspase activation in retinas of diabetic and galactosemic mice and diabetic patients. Diabetes 2002;51:1172-1179.

50 Park HY, Kim JH, Park CK: Neuronal cell death in the inner retina and the influence of vascular endothelial growth factor inhibition in a diabetic rat model. Am J Pathol 2014;184:1752-1762.

51 Kern TS, Barber AJ: Retinal ganglion cells in diabetes. J Physiol 2008;586:4401-4408.

52 Zhou RM, Shen Y, Yao J, Yang H, Shan K, Li XM, Jiang Q, Yan B: Nmnat 1: a Security Guard of Retinal Ganglion Cells (RGCs) in Response to High Glucose Stress. Cell Physiol Biochem 2016;38:2207-2218.

53 Whibley N, Tritto E, Traggiai E, Kolbinger F, Moulin P, Brees D, Coleman BM, Mamo AJ, Garg AV, Jaycox JR, Siebenlist U, Kammuller M, Gaffen SL: Antibody blockade of IL-17 family cytokines in immunity to acute murine oral mucosal candidiasis. J Leukoc Biol 2016;99:1153-1164.

54 Russell CB, Rand H, Bigler J, Kerkof K, Timour M, Bautista E, Krueger JG, Salinger DH, Welcher AA, Martin DA: Gene expression profiles normalized in psoriatic skin by treatment with brodalumab, a human antiIL-17 receptor monoclonal antibody. J Immunol 2014;192:3828-3836.

55 Ke Y, Liu K, Huang GQ, Cui Y, Kaplan HJ, Shao H, Sun D: Anti-inflammatory role of IL-17 in experimental autoimmune uveitis. J Immunol 2009;182:3183-3190.

-56 Fletcher EL, Downie LE, Ly A, Ward MM, Batcha AH, Puthussery T, Yee P, Hatzopoulos KM: A review of the role of glial cells in understanding retinal disease. Clin Exp Optom 2008;91:67-77.

57 Kim EL, Moshfeghi AA: Effect of Intravitreal Anti-VEGF Therapy on the Severity of Diabetic Retinopathy. Curr Ophthalmol Rep 2016;4:61-70.

58 Li J, Wang P, Ying J, Chen Z, Yu S: Curcumin Attenuates Retinal Vascular Leakage by Inhibiting Calcium/ Calmodulin-Dependent Protein Kinase II Activity in Streptozotocin-Induced Diabetes. Cell Physiol Biochem 2016;39:1196-1208.

-59 Ishimoto Y, Hirota-Takahata Y, Kurosawa E, Chiba J, Iwadate Y, Onozawa Y, Hasegawa T, Tamura A, Tanaka M, Kobayashi H: A Novel Natural Product-Derived Compound, Vestaine A1, Exerts both Pro-Angiogenic and Anti-Permeability Activity via a Different Pathway from VEGF. Cell Physiol Biochem 2016;39:1905-1918. 